\title{
Low Complexity Cyclostationary Feature Detection Method to Compenstate Cyclostationarity- Degradation by Guard Interval Insertion
}

\author{
${ }^{\dagger}$ Hiroyoshi Yano, ${ }^{\dagger}$ Osamu Takyu, ${ }^{\dagger}$ Takeo Fujii, and ${ }^{\dagger}$ Tomoaki Ohtsuki \\ 'School of Science for OPEN and Environmental Systems \\ Keio University \\ yano@nkgw.ics.keio.ac.jp, ohtsuki@ics.keio.ac.jp \\ "Electrical and Electronic \\ Advanced Wireless Comm. Research Centre \\ Engineering, Shinshu University University of Electro-Communications \\ takyu@shinshu-u.ac.jp $\quad$ fujii@awcc.uec.ac.jp
}

\begin{abstract}
In cognitive radio system, it is necessary for spectrum sensing technology to search for vacant channel. Cyclostationary feature detection method has superior noise-immune and recognition. However, since the mismatch between the detected signal and detection duration by guard interval (GI) insertion occurs, the cyclostationarity is degraded. In this paper, we propose a high accuracy and low complexity detection method based on cyclostationarity feature. The proposed method limits symbol detection time and makes multiple delay line detectors as units of the GI time. Computer simulation and analytical evaluation show the effectiveness of the proposed detection method compared to the conventional one.
\end{abstract}

\section{INTRODUCTION}

To efficiently utilize the precious radio spectrum, a cognitive radio (CR) has been considered as a key technology. In $\mathrm{CR}$, the secondary system (SS) actively uses the available frequency resources to highly control the variable parameters, such as transmit power and frequency bandwidth, and not to give serious interference the primary system (PS). In CR, PS does not always notify the usage of the frequency resources. Therefore, SS needs a signal detection technique to recognize that PS uses frequency resources or not [1], [2].

Cyclostationary feature detection method is one of the most powerful methods [2], [3]. In this method, the receiver obtains the feature components based on the spectrum correlation function (SCF) from the detected signal [2]. SCF is composed of the frequency of sinusoidal wave and the cyclic frequency. In double dimension, frequency and cyclic frequency, the original peak patterns for each modulated symbol appear.

Recently, broadband wireless systems adopt a multi-carrier modulation technique, such as orthogonal frequency division multiplexing (OFDM). In multi-carrier transmission systems, for estimating the frequency offset, the pilot signal is inserted to the particular subcarriers. We can obtain high correlation peak components (SCF peak) from the pilot signal [6]. The high accurate detection can be achieved by high SCF peak even under the low signal to noise power ratio (SNR) [5] - [7]. In broadband wireless systems, for avoiding inter-symbol interference (ISI) in multi-path environment, guard interval (GI) is pre-inserted to the transmitted signal. By the insertion of GI, however, the detection signal becomes discontinuous and therefore cyclostationarity of pilot signal is degraded.
Ref. [7] derives a new formula for SCF of OFDM signals. This formula can be used as the compensator for the cyclostationary feature degraded by GI insertion. However, this method not only enlarges the computational complexity but also degrades the noise immunity because of spreading SCF peaks around several cyclic frequencies.

In this paper, we propose a low-complexity cyclostationary feature detection method for compensating cyclostationaritydegradation by GI insertion. In the proposed method, the detection duration is matched to the OFDM symbol one. In addition, for avoiding the gap between the detection duration and the OFDM symbol, we set the period where we stop detecting. As a result, it is avoidable for the detected signal to be discontinuous. Furthermore, in order to avoid discontinuous detection due to symbol timing error under the asynchronous environment, we propose multi-tap delay line filter whose unit delay is equal to GI length. The timing error of the detected signal in at least one of lines is less than GI length. If more highly accurate timing detection is desirable, the tap delay should be equal to minimum sample duration but computational complexity becomes much larger. We confirm the constructed filter is enough to achieve the accurate detection. From the computer simulation, we show that the proposed method achieves the accurate detection due to compensating the cyclostationarity-degradation by GI insertion under the low computational complexity.

The rest of the paper is organized as follows. In Section II we present a background of this research, and in Section III we apply the SCF calculation to IEEE802.11. In Sections IV and V, we present the conventional and proposed method, respecttively. Section VI shows simulation results and discussion and Section VII shows the result of comparison complexity. We finally provide some concluding remarks in Section VIII.

\section{BACKGROUND}

In this section, we first show assumed wireless communication and then we derive second-order cyclostatonarity for SCF.

\section{A. System Assumption}

We consider the target system adopts the standard of IEEE 802.11. For simple explanation, we refer to the target system as primary system (PS). We also assume the cognitive radio's terminal which tries to detect PS's accessing is secondary system (SS). Carrier sense multiple access (CSMA) has a sensing function, simple energy detection. For example, a threshold of IEEE802.11 is $-62 \mathrm{dBm}$ signal level above the minimum. 
Therefore, CSMA cannot detect signal near or below the noise level. Then, a hidden terminal problem exists. Therefore, if SS also uses CSMA, it cannot detect a feeble signal. We assume that PS is in communication with base station (BS) and SS senses PS signal but a hidden terminal problem occurs between PS and SS. Thus SS needs a high-performance sensing to detect a feeble signal and recognizes the system.

\section{B. Concept of Second-Order Cyclostatonarity for SCF}

Let $x[i]$ be a complex discrete time signal. The cyclic autocorrelation function (CAF) for complex discrete time signal $x[i]$, is defined as follows [5]

$$
\tilde{R}_{x}[\gamma, \alpha]=\frac{1}{I_{0}} \sum_{i=i_{0}}^{i_{0}+I_{0}-1} x[i] x^{*}[i+\gamma] e^{-j 2 \pi \alpha i T_{S}}
$$

where $*$ denotes conjugation, $I_{0}$ is the observation interval, $i_{0}$ is the starting observation sample point and $T_{S}$ is the sampling time. When $\tilde{R}_{x}[\gamma, \alpha] \neq 0$ for $\alpha \neq 0, \alpha$ is the cyclic frequency of $x[i]$ at lag parameter $\gamma$, and $x[i]$ is said to exhibit secondorder cyclostationarity.

For using the Fourier transform, $v$-th OFDM complex symbol on time domain $x_{v}[i]$ can be expressed by

$$
x[i]=\sum_{i=0}^{N_{d}-1} X[f] e^{j 2 \pi f i T_{S}}
$$

where $N_{d}$ is the size of the fast Fourier transform (FFT) used. By substituting eq. (2) into (1),

$$
\begin{aligned}
& \tilde{R}_{x}[\gamma, \alpha] \\
& =\frac{1}{I_{0}} \sum_{i=i_{0}}^{i_{0}+I_{0}-1} \sum_{f_{1}} \sum_{f_{2}} X\left[f_{1}\right] X^{*}\left[f_{2}\right] e^{j 2 \pi\left(f_{1}-f_{2}-\alpha\right) i T_{s}} e^{-j 2 \pi f_{2} \gamma T_{s}} .
\end{aligned}
$$

Second-order cyclostationarity gives rise to the specific correlation patterns which occur in the spectrum of the signal. These patterns can be analyzed using the SCF, and it is defined as follows [2]

$$
\tilde{S}_{x}[f, \alpha]=\sum_{\gamma=0}^{\Gamma} R_{x}[\gamma, \alpha] e^{-j 2 \pi f \gamma T_{s}}
$$

where $f$ is frequency, $\Gamma$ is the observation lag parameter. Substituting eq. (4) into (3), if we derive $f=f_{1}-\alpha$, it is expressed as follows

$$
\begin{aligned}
& \tilde{S}_{x}[f, \alpha] \\
& =\frac{1}{I_{0}} \sum_{\gamma=0}^{\Gamma} \sum_{i=i_{0}}^{i_{0}+I_{0}-1} \sum_{f_{1}} \sum_{f_{2}} X\left[f_{1}\right] X^{*}\left[f_{2}\right] e^{j 2 \pi\left(f_{1}-f_{2}-\alpha\right)(i-\gamma) T_{s}} .
\end{aligned}
$$

Here, when $I_{0}$ and $\Gamma$ approach infinity,

$$
\frac{1}{I_{0}} \sum_{\gamma=0}^{\Gamma} \sum_{i=i_{0}}^{i_{0}+I_{0}-1} e^{j 2 \pi\left(f_{1}-f_{2}-\alpha\right)(i-\gamma) T_{s}}=\left\{\begin{array}{lc}
1, & f_{1}-f_{2}-\alpha=\frac{Z}{T_{S}} \\
0, & \text { otherwise }
\end{array}\right.
$$

where $z$ is $\quad z \in \boldsymbol{Z}$ ( $\boldsymbol{Z}$ is the integer set). Therefore, eq. (6) becomes non-zero only at $\alpha=f_{1}-f_{2}-z / T_{s}$. On the other hand, from eq. (1), the CAF for $\alpha=\alpha_{0}$ and that for $\alpha=\alpha_{0}+$ $a / T_{s}(a \in \mathbf{Z})$ are same. Therefore, we can simply focus on the

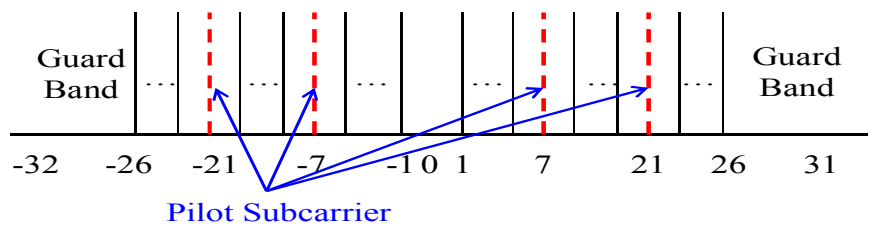

Fig 1. IEEE802.11 standard subcarrier allocation

case of $z=0$. Accordingly, when $I_{0}$ and $\Gamma$ approach infinity, eq. (5) can be rewritten as

$$
\tilde{S}_{x}[f, \alpha]=X[f] X^{*}[f-\alpha] .
$$

\section{CyClOSTATIONARY METHOD FOR IEEE 802.11}

This section shows typical expression of SCF in the OFDM signal, IEEE802.11 a/g standards.

\section{A. OFDM Signal in SCF}

Let $X_{v}[f]$ be a $v$-th OFDM complex symbol on frequency domain. The SCF for OFDM signal is expressed as follows

$$
\tilde{S}_{x}[f, \alpha]=\frac{1}{V} \sum_{v=0}^{V-1} X_{v}[f] X_{v}{ }^{*}[f-\alpha]
$$

where $V$ is the number of observation symbols. When $\tilde{S}_{x}[f, \alpha] \neq 0$ for $\alpha \neq 0, \alpha$ is the cyclic frequency of $X_{v}[f]$ at frequency $f$, and $X_{v}[f]$ is said to exhibit second-order cyclostationarity.

For using a inverse FFT, $v$-th OFDM complex symbol in time domain $x_{v}[i]$ can be expressed

$$
X_{v}[f]=\sum_{i=0}^{N_{d}-1} x_{v}[i] e^{-j 2 \pi f i T_{s}} .
$$

\section{B. SCF Peak Appeared Position for IEEE802.11 Standard}

On IEEE802.11 a/g standards [8], the $N_{d}=64$-point IFFT is used with a carrier spacing of $\Delta f=312.5 \mathrm{kHz}$. This leads to a time duration of one IFFT output symbol of $T_{s}=3.2 \mu \mathrm{s}$. The GI is of duration $T_{g}=0.8 \mu \mathrm{s}$. From the above definitions, one OFDM symbol is of duration $T=T_{s}+T_{g}=4.0 \mu \mathrm{s}$ and $T_{g}=T_{s} / 4$. Each subcarrier $f_{k}$ is expressed as $f_{k}=k \Delta f=$ $k / T_{s}(k \in[-32,31])$, where $k$ is the number of OFDM subcarriers. Also $k=\{-32, \ldots,-27,27, \ldots, 31\}$ th subcarriers remain unused in order to construct guard bands. The $0^{\text {th }}$ subcarrier, $k=0$, is excluded in order to avoid a DC offset. In addition, as shown in Fig $1, k=\{-21,-7,7,21\}$ th subcarriers have been used as pilot signal and called pilot subcarrier. In order to avoid the formation of spectral lines, the pilot signal is two-valued pseudo-random sequence of BPSK modulated. The phase of the 21th subcarrier is inverted, but these phase rotational rate are equal [8]. Here, if $f_{1}, f_{2}$ described in Section II are pilot subcarriers respectively, the SCF shows SCF peaks for

$$
\left\{\begin{array}{l}
f=f_{1}-\alpha \\
\alpha=f_{1}-f_{2}-z / T_{s}
\end{array}\right.
$$



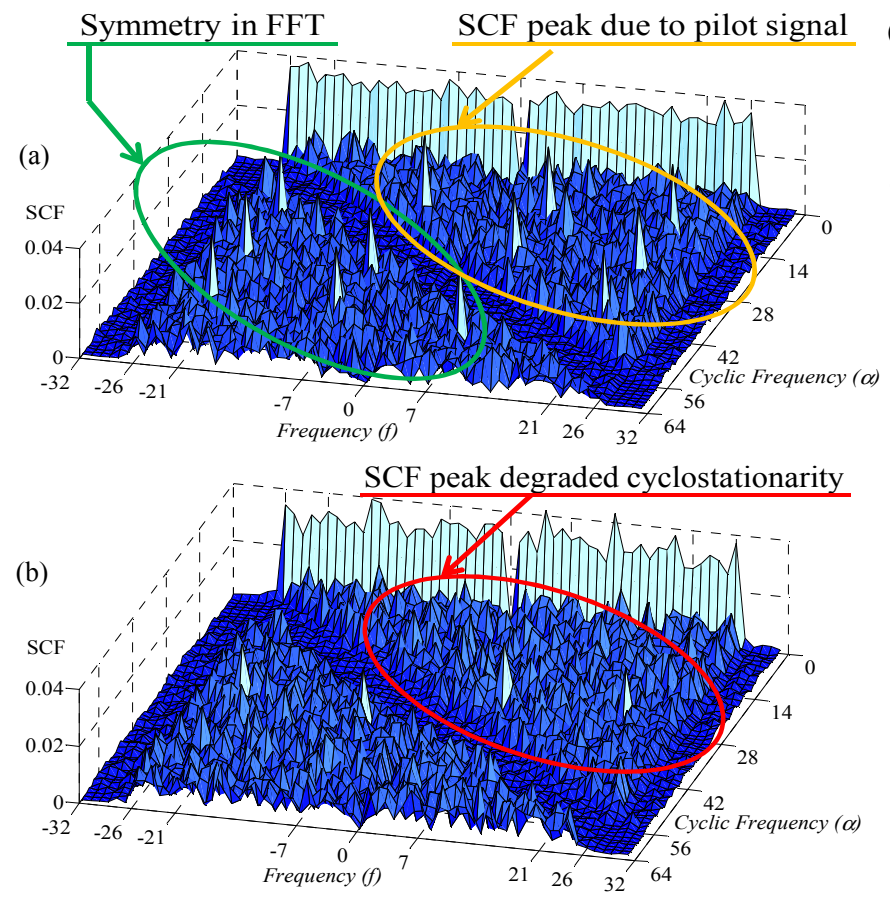

Fig 2. $\quad$ SCF of IEEE802.11 (a) w/o GI (b) with GI

where $z$ is $\quad z \in \boldsymbol{Z}$ ( $\boldsymbol{Z}$ is the integer set). Fig 2 (a) shows an example of the output of the SCF. As four pilot subcarriers are used, there are $2 \times\left(\begin{array}{l}4 \\ 2\end{array}\right)=12$ peaks in total, however six peaks are caused by the symmetry in FFT.

Next, we consider the transmitted signal when GI was inserted. SCF peak appeared condition in case of being inserted GI is derived as the following equation [7].

$$
\tilde{S}_{x}[f, \alpha]= \begin{cases}1, & \left(f_{1}-f_{2}\right) T_{g} \in \boldsymbol{Z} \\ 0, & \text { otherwise }\end{cases}
$$

By the insertion of the GI, some peaks not to satisfy the eq. (11) exists. For instance, for $f_{1}=7 / T_{s}, f_{2}=-7 / T_{s}$ we have $\left(f_{1}-f_{2}\right) T_{g}=3.5 \notin Z$. Thus, there is no peak at $(f, \alpha)=$ $(-7,14)$. Fig 2 (b) shows an example of the output of the SCF for GI insertion. From the figure, we can see that the number of peaks decreasesand the power of each SCF peak becomes small. Therefore, we confirm the cyclostationarity-degradation.

\section{METHOD FOR COMPENSATING CYCLOSTATIONARITY- DEGRADATION BY GUARD INTERVAL INSERTION \\ (CONVENTIONAL METHOD)}

Ref. [7] gives a new formula for the SCF of OFDM signals. In this formula, the cyclic frequency becomes higher resolution and thus the disappeared SCF peak is detectable. We can consider this formula is available for compensating the cyclostationarity-degradation, so that this is the conventional method. In the conventional method, it defines $\alpha^{\prime}$ such as eq. (12) in order to compensate for peaks.

$$
\begin{array}{r}
\alpha^{\prime}=d^{\prime} / \operatorname{LCM}\left(T_{s}, T\right)=\left(\operatorname{LCM}\left(T_{s}, T\right) / T_{s}\right) \times \alpha, \\
d^{\prime} \in \boldsymbol{Z}, d^{\prime} \in[0,320]
\end{array}
$$

where the operator $\operatorname{LCM}(a, b)$ denotes the least common multiple of $a$ to $b$. In IEEE $802.11 \mathrm{a} / \mathrm{g}$ standard, $\alpha^{\prime}$ is five times higher resolution of $\alpha$ [7]. As a result, the amount of SCF (a)

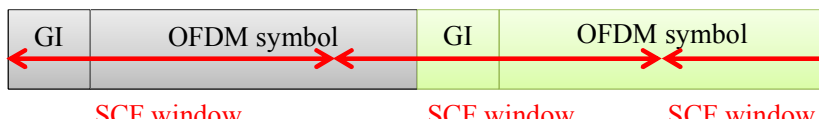

(b)

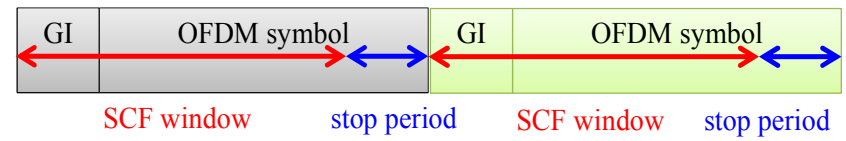

Fig 3. (a) Conventional SCF calculation method, (b) proposed SCF calculation method

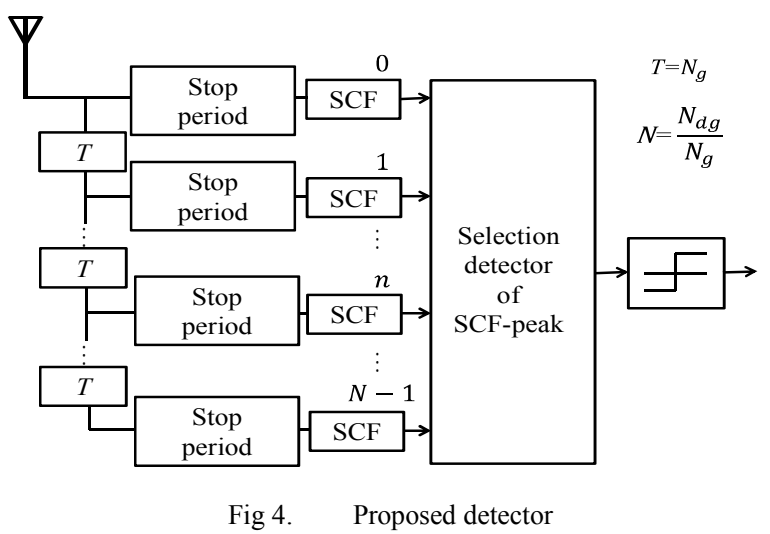

sated peak is divided into two parts. Therefore, the power per single peak is lower than that in the case without GI insertion so this method is less robustness for noise.

\section{PROPOSED METHOD}

The calculation of SCF separates received signal into some detection windows (SCF window) corresponding to the OFDM symbol interval, and calculates each SCF window. In the conventional detection method, Fig 3 (a) shows the relationship between OFDM symbol and detection window. We can see the mismatch between the SCF window and OFDM symbol becomes larger due to the insertion of GI. In the proposed method, the stop period is inserted after OFDM symbol duration such as Fig. 3 (b). As a result, we can avoid the increase of timing mismatch between detected symbol and OFDM symbol. If detection timing error is less than GI length, we can avoid a discontinuous detection. Thus, we can avoid cyclostationarity-degradation.

However, detection timing error over GI length degrades cyclostionarity, significantly. Therefore, we also propose multitap delay line filter. Fig 4 shows the block diagram of the proposed detector, where the taps delay period is equal to GI length. Furthermore, since each cyclostationary detector may be calculated in integer intervals. In the proposed detector, at least one of parallel processing lines is able to detect the signal with the smaller timing error signal than GI length. After that, each result of parallel line process is put into the selection process. In the selection process, the result of the largest SCF peak is selected. In the proposed detector, the number of parallel process lines is determined by the length of the transmitted signal to the GI symbol length ratio and is expressed as follows

$$
N=\frac{N_{d g}}{N_{g}}
$$


TABLE I. SimUlation PALAMETER

\begin{tabular}{|c|c|}
\hline Modulation & QPSK \\
\hline FFT size, GI length & 64,16 \\
\hline Number of subcarriers & $\begin{array}{c}\text { DATA: } 48 \\
\text { Pilot: } 4\end{array}$ \\
\hline Number of observation symbols $V$ & 16,8 to 32 \\
\hline $\begin{array}{c}\text { Starting observation } \\
\text { sample point } i_{0}\end{array}$ & $\begin{array}{c}i_{0} \in[0,79], \\
\text { uniform distribution }\end{array}$ \\
\hline Threshold setting & Miss Detection $1 \%$ \\
\hline Channel model & $\begin{array}{c}\text { i) AWGN channel } \\
\text { ii) Equal power 8-path Rayleigh } \\
\text { fading channel } \\
\left.\text { (Max. Doppler freq., } f_{D} \cong 0 \mathrm{~Hz}\right)\end{array}$ \\
\hline
\end{tabular}

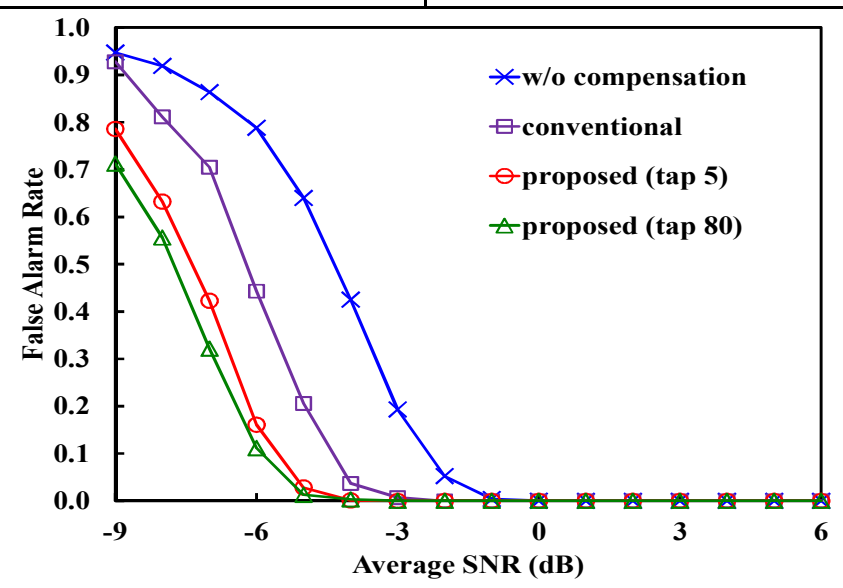

Fig 5. False alarm rate vs Average SNR under AWGN condition

where $N$ is the number of parallel process lines. For example, in IEEE $802.11 \mathrm{a} / \mathrm{g}$ and WiMax standard, the required number, of parallel process lines are 5 and 9 , respectively.

\section{Simulation Results}

From the computer simulation, we evaluate the detection accuracy of proposed method. Table 1 shows the simulation parameters in IEEE802.11 a/g standard.

The PS signal detection problem can be modeled as twovalued binary hypotheses test as follows. Miss detection is that despite PS using a frequency channel, detector judges "idle". On the contrary, false alarm is that despite PS not using a frequency channel, detector judges "busy". In this paper, we set a detection threshold as miss detection probability (MDP) is $1 \%$, and then we evaluate false alarm rate (FAR).

We consider two conventional methods. First one is the cyclostationary feature detection without compensating cyclostationarity-degradation. Second one is that with compensating the cyclostationarity-degradation by fractional frequency [7]. First method and second one are "w/o compensation" and "conventional", respectively. In proposed method, we consider two kinds of tap delay period in proposed detector. First one is GI length and second one is minimum sample duration. In the former and latter, the number of parallel process lines, $N$ are 5 and 80 , respectively and thus "proposed (tap 5)" and

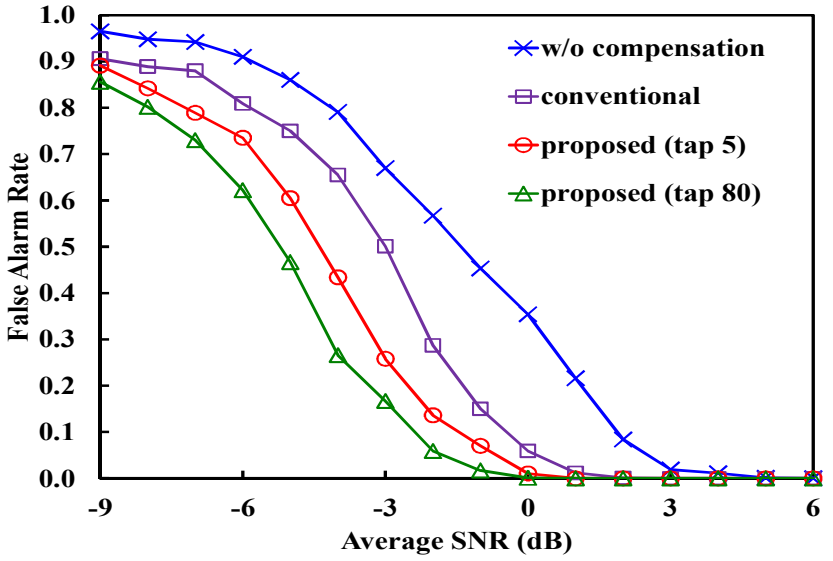

Fig 6. False alarm rate vs Average SNR under multipath fading channel

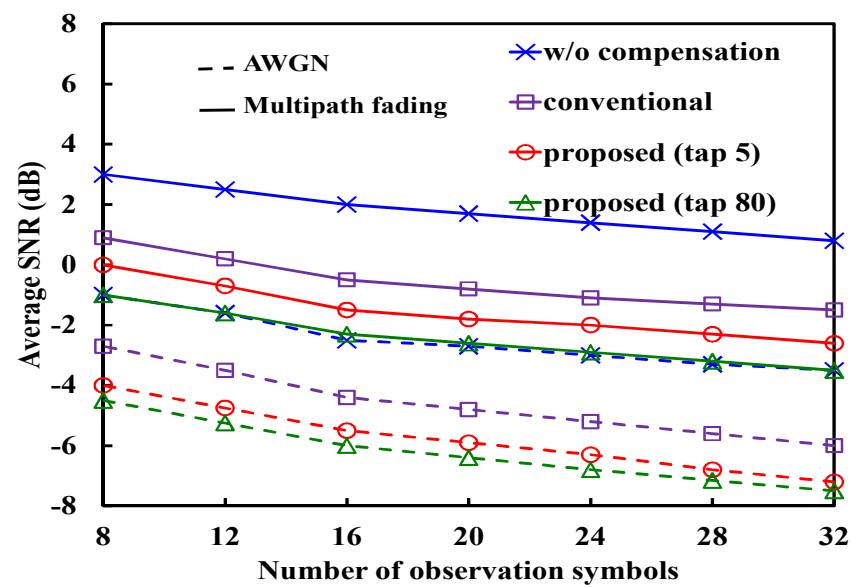

Fig 7. Average SNR vs number of observed symbolsl

"proposed (tap 80)", respectively

\section{A. FAR vs Average SNR on AWGN Channel}

Fig. 5 shows FAR as a function of the average SNR under AWGN channel. We can see that the required SNRs for achieving $10 \%$ false alarm are about $-2.5,-4.5,-5.5,-6 \mathrm{~dB}$, "w/o compensation", "conventional", "proposed (tap 5)" and "proposed (tap 80)", respectively. Accordingly, the required SNR can be reduced for $1.5 \mathrm{~dB}$ by the proposed (tap 5) and $2 \mathrm{~dB}$ by the proposed (tap 80 ), respectively, because the proposed method does not divide compensated SCF peaks Therefore, it is shown that the proposed method is more effective to compensate cyclostationarity-degradation.

\section{B. FAR vs Average SNR on Multipath Rayleigh Fading Channel}

Fig 6 shows FAR as a function of the average SNR on multipath Rayleigh fading channel. We can see that the required SNRs for achieving $10 \%$ false alarm are about $2,-0.5,-1.5,-2.5 \mathrm{~dB}$, “w/o compensation", "conventional", "proposed (tap 5)" and "proposed (tap 80)", respectively. Accordingly, the required SNR can be reduced for $1 \mathrm{~dB}$ by the proposed (tap 5) and $2 \mathrm{~dB}$ by the proposed (tap 80), respectively. However, to compare the proposed (tap 5) with the proposed (tap 80), the proposed (tap 5) increases the required 
TABLE II. EXAMPLE OF EACH MODEL'S PALAMETER AND COMPLEXITY

\begin{tabular}{|c|c|c|c|c|}
\hline & $N$ & $L$ & $\alpha$ & complexity $(C)$ \\
\hline w/o compensation & 1 & 80 & 64 & 327680 \\
\hline conventional & 1 & 80 & 320 & $327680 \times 25$ \\
\hline proposed (tap 5) & 5 & 64 & 64 & $327680 \times 4$ \\
\hline proposed (tap 80) & 80 & 64 & 64 & $327680 \times 64$ \\
\hline
\end{tabular}

SNR for $1 \mathrm{~dB}$ because ISI occurs. Since the proposed (tap 5) method sets the tap length to GI length, temporal resolution is low. As a result, it cannot detect first path, and thus ISI occurs and cyclostationarity degrades. On the other hand, the proposed (tap 80) can detect the first path accurately owing to parallel processes with the high temporal resolution.

\section{Average SNR vs Number of Observed Symbols}

Fig. 7 shows average SNR vs the number of observed symbols with MDP $=1 \%$ and FAR $=10 \%$. We know that all detection methods are robust to AWGN at the cost of increasing observation symbols, because SCF peak due to AWGN vanishes as the observation symbols is infinity. In CR, however, SS wants to reduce the number of observation symbols because of the effective use of vacant time. The required the number of observed symbols can be reduced for 24 symbols by achieving SNR $-2 \mathrm{~dB}$ by the proposed (tap 5).

\section{COMPARISON WITH CONVENTIONAL METHODS IN TERMS OF COMPLEXITY}

In this section, we analytically evaluate the computational complexity of four methods. The parameters are based on Table I described in Section VI. In the detection method of all, SCF formula of OFMD symbol from eq. (1) and (4) is as follows:

$\tilde{S}_{x}[n, f, \alpha]=\frac{1}{L} \sum_{\gamma=0}^{\Gamma} \sum_{i=i_{0}}^{i_{0}+L-1} x[i] x^{*}[i+\gamma] e^{-j 2 \pi(\alpha i+f \gamma) T_{S}}$

where $L$ is calculating length per OFDM symbol and $n$ is the number of parallel processes. Here, we focus on three parameters, $n, i$ and $\alpha$. In this paper, because the multiplication process dominates the amount of calculation as shown in eq. (14), the number of multiplication is expressed as the overall complexity. Because each detection method has various numbers of these three parameters, we only evaluate these parameters. Therefore, the formula of complexity is as follows:

$$
C=(L \times \alpha) \times \alpha \times N
$$

where $C$ is the number of multiplication, that indicates complexity, and $N$ is the number of parallel process lines.

The numerical examples of each detection method are shown in Table II. As shown in complexity in Table II, the relative complexity between w/o compensation method and other methods. It is shown that the complexity of the proposed (tap 5) method is $1 / 6$ smaller than that of the conventional method. The reason why the conventional method needs the huge complexity is that the cyclic frequency is fractional intervals. The proposed technique uses the SCF calculation of integer cyclical frequency and thus the complexity is small. In addition, it is shown that the complexity of the proposed (tap 5) method is $1 / 16$ smaller than of the proposed (tap 80) method. The reason why the proposed (tap 5) reduces parallel processes is that it changes tap length to GI length. In summary, the proposed (tap 5) method can reduce the complexity to $1 / 16$ but increases the SNR to achieve the FAR of $10 \%$ about $1 \mathrm{~dB}$ in Multipath fading environment, which shows that tradeoff between the complexity and the detection performance exists. On the other hand, the proposed (tap 5) method compared to the conventional method can reduce the complexity to around $1 / 6$ and the SNR to achieve the FAR of $10 \%$ about $2 \mathrm{~dB}$ in Multipath fading environment.

\section{CONCLUSIONS}

In this paper, we proposed detection method with high accuracy and low complexity for spectrum sensing based on the cyclostationarity-induced pilot signal degraded by GI insertion. The proposed method adds stop period equal to GI length and avoids the increase of timing mismatch between detected symbol and detection window. Moreover, in order to avoid cyclostationarity-degradation by synchronization of timing shift, we propose multi-tap delay line filter whose unit delay is equal to GI length.

As a result of evaluating false alarm rate by computer simulation, we showed that the proposed method achieves lower false alarm rate than the conventional one. By analytical evaluation, we also showed that the proposed method can reduce the amount calculation than the conventional one.

\section{ACKNOWLEDGEMENT}

A part of this research project is sponsored by Ministry of Internal Affairs and Communications in Japan as a project name of Strategic Information and Communications R\&D Promotion Programme (SCOPE 092103009).

\section{REFERENCES}

[1] S.S.Hwan, H.Ning, K.J.Moung and K.J.Wan, "OFDM Signal Sensing Method Based on Cyclostaionary Detection," Cognitive Radio Oriented Wireless Networks and Communications(CrownCom), pp.63-68, Aug. 2007

[2] P.D.Sutton, K.E.Nolan and L.E.Doyle, "Cycostaionary Signatures in Practical Cognitive Radio Appli- cations," IEEE Journal on Selected Areas in Communications (JSAC), Vol.26, no.1, pp.13-24, Jan. 2008.

[3] D. Cabric, S. M. Mishra, and R. W. Brodersen, "Implementation issues in spectrum sensing for cognitive radios," Proc. Asilomar Conference on Signals, Systems and Computers, Vol.1, pp.772-776, Nov. 2004.

[4] Z.Lei and F.Chin "OFDM Signal Sensing for Cognitive Radios," Personal, Indoor and Mobile Radio Communications (PIMRC), pp.1-5, Sept. 2008

[5] K.Maeda, A.Benjebbour, T.Asai, T.Furuno and T.Ohya, "Cyclostationarity-inducing transmission methods for recognition among OFDMbased systems," EURASIP Journal on Wireless Communications and Networking, vol.2008, Article ID 586172, 14 pages, 2008.

[6] S.Kandeepan, G.Baldini, R.Piesiewicz, "Experimentally Detecting IEEE $802.11 \mathrm{n}$ Wi-Fi Based on Cyclostationarity Features for Ultara-Wide Band Cognitive Radios," Personal, Indoor and Mobile Radio Communications (PIMRC), pp.2315-2319, Sept. 2009

[7] M. adrat, J.Lrduc, S.Couturier, M.Antweiler, H.E.Boll, "2 ${ }^{\text {nd }}$ Order Cyclostationarity of OFDM Signals: Impact of Pilot tones and Cyclic Prefix," IEEE International Conference (ICC), pp.14-18, June. 2009

[8] IEEE Standard for Information technology, Telecommunications and information exchange between systems, Local and metropolitan area networks, Specific requirements, Part 11: 